\title{
Linear algebraic theory of partial coherence: continuous fields and measures of partial coherence
}

\author{
Haldun M. Ozaktas, ${ }^{1}$ Talha Cihad Gulcu, ${ }^{2, *}$ and M. Alper Kutay ${ }^{3}$ \\ ${ }^{1}$ Department of Electrical Engineering, Bilkent University, TR-06800 Bilkent, Ankara, Turkey \\ ${ }^{2}$ Department of Electrical and Computer Engineering, University of Maryland, College Park, Maryland 20742, USA \\ ${ }^{3}$ The Scientific and Technological Research Council of Turkey, Ataturk Bulvari 221, TR-06100 Kavaklidere, Ankara, Turkey \\ *Corresponding author: tcgulcu@gmail.com
}

Received 8 July 2016; revised 2 September 2016; accepted 2 September 2016; posted 7 September 2016 (Doc. ID 269808);

published 4 October 2016

\begin{abstract}
This work presents a linear algebraic theory of partial coherence for optical fields of continuous variables. This approach facilitates use of linear algebraic techniques and makes it possible to precisely define the concepts of incoherence and coherence in a mathematical way. We have proposed five scalar measures for the degree of partial coherence. These measures are zero for incoherent fields, unity for fully coherent fields, and between zero and one for partially coherent fields. () 2016 Optical Society of America
\end{abstract}

OCIS codes: (030.0030) Coherence and statistical optics; (030.1640) Coherence.

http://dx.doi.org/10.1364/JOSAA.33.002115

\section{INTRODUCTION}

The theory of partially coherent optical fields is well established [1-23]. In a previous work, where we concentrated on discrete fields [24-26], we saw that new perspectives can be gained by reformulating the theory based on linear algebraic concepts. This approach brings new understanding, insight, and operationality and may be useful in applications such as optical signal processing. Several of the concepts proposed in [24] have been used or referred to in works published since then [14,27-30].

In this work we considered optical fields of continuous variables. While the discrete fields considered in [24] led to a matrix-algebraic formulation, the case of continuous fields leads to a formulation in continuous function spaces. We assumed quasi-monochromatic conditions and do not deal with temporal coherence effects. We focused on the degree of spatial coherence of light fields, as quantified by their autocorrelation, or mutual intensity functions.

The conventional second-order theory of spatial coherence associated with paraxial propagation is presented in [4]. In the case of micro-diffraction, however, paraxial theories are unable to predict some phenomena, and non-paraxial theories are more suitable. The tensor theory of [31] is an example of such a theory.

We first defined several functions that quantify secondorder correlations. Then we used these functions to define full incoherence and full coherence. Following that, we have proposed five scalar measures for the degree of partial coherence of a field, which vary from 0 for the fully incoherent case, to 1 for the fully coherent case. The section before the conclusion discusses some of the concepts in the Fourier domain.

\section{MUTUAL INTENSITY FUNCTION}

Let the function $f(x)$ be a random process representing a random optical field. We will deal with one-dimensional signals for simplicity, but extending the results to two dimensions is not difficult. We can define the mutual intensity function $J_{f}\left(x_{1}, x_{2}\right)$ of $f(x)$ as

$$
J_{f}\left(x_{1}, x_{2}\right)=\left\langle f\left(x_{1}\right) f^{*}\left(x_{2}\right)\right\rangle .
$$

Here the angular brackets represent the ensemble average (expectation value). The raised $*$ represents the complex conjugate. We drop the subscript $f$ in $J_{f}\left(x_{1}, x_{2}\right)$ and simply write $J\left(x_{1}, x_{2}\right)$ if there is no possibility of confusion. The intensity is given by $J(x, x)=I(x)$ and the power is given by $\int I(x) \mathrm{d} x$. Unless indicated otherwise, integrals extend over the interval on which $f$ is defined. The mean-subtracted mutual intensity, denoted $K_{f}$, is defined as

$$
K_{f}\left(x_{1}, x_{2}\right)=\left\langle\left[f\left(x_{1}\right)-\mu_{f}\left(x_{1}\right)\right]\left[f\left(x_{2}\right)-\mu_{f}\left(x_{2}\right)\right]^{*}\right\rangle,
$$

where $\mu_{f}(x)=\langle f(x)\rangle$ is the mean of $f(x)$, a function whose value at each point is the mean of $f(x)$ at that point. We noted that $K(x, x)=\sigma^{2}(x)$ is the variance of the field. In the language of the theory of random processes or statistics, the mutual intensity $J\left(x_{1}, x_{2}\right)$ is an autocorrelation function whereas 
its mean-subtracted version $K\left(x_{1}, x_{2}\right)$ is an autocovariance function. The relation between $J\left(x_{1}, x_{2}\right)$ and $K\left(x_{1}, x_{2}\right)$ is given by $J\left(x_{1}, x_{2}\right)=K\left(x_{1}, x_{2}\right)+\mu_{f}\left(x_{1}\right) \mu_{f}^{*}\left(x_{2}\right)$.

$J\left(x_{1}, x_{2}\right)$ and $K\left(x_{1}, x_{2}\right)$ satisfy the following properties (the proofs are omitted where elementary or well-known [32]):

1. $J\left(x_{1}, x_{2}\right)$ and $K\left(x_{1}, x_{2}\right)$ are Hermitian symmetric: $J\left(x_{1}, x_{2}\right)=J^{*}\left(x_{2}, x_{1}\right)$ and $K\left(x_{1}, x_{2}\right)=K^{*}\left(x_{2}, x_{1}\right)$. This follows directly from the definitions of $J\left(x_{1}, x_{2}\right)$ and $K\left(x_{1}, x_{2}\right)$.

2. Since they are Hermitian-symmetric functions, $J\left(x_{1}, x_{2}\right)$ and $K\left(x_{1}, x_{2}\right)$ have real eigenvalues. The eigenvalue equation for $J\left(x_{1}, x_{2}\right)$ is

$$
\int J\left(x, x^{\prime}\right) u_{k}\left(x^{\prime}\right) \mathrm{d} x^{\prime}=\lambda_{k} u_{k}(x),
$$

where $\lambda_{k}$ and $u_{k}(x)$ are the eigenvalue and eigenfunction with index $k$.

3. $J\left(x_{1}, x_{2}\right)$ and $K\left(x_{1}, x_{2}\right)$ are positive semi-definite, and as a consequence, the eigenvalues are non-negative. Positive semi-definiteness is expressed as

$$
\iint u^{*}\left(x_{1}\right) J\left(x_{1}, x_{2}\right) u\left(x_{2}\right) \mathrm{d} x_{1} \mathrm{~d} x_{2} \geq 0
$$

for any function $u(x)$. Furthermore, $\left|J\left(x_{1}, x_{2}\right)\right|^{2} \leq\left|J\left(x_{1}, x_{1}\right)\right| \mid$ $J\left(x_{2}, x_{2}\right) \mid$, and likewise for $K\left(x_{1}, x_{2}\right)$.

4. As is the case for all Hermitian-symmetric functions, eigenfunctions with different eigenvalues must be orthogonal. Moreover, a set of orthonormal eigenfunctions always exists even when there are degenerate eigenvalues. We assumed the set of eigenfunctions is chosen to be orthonormal. Mathematically, orthonormality is expressed as

$$
\int u_{k}^{*}(x) u_{k^{\prime}}(x) \mathrm{d} x=\delta_{k k^{\prime}},
$$

where $\delta_{k k^{\prime}}$ is the Kronecker delta (i.e., $\delta_{k k^{\prime}}=1$ when $k=k^{\prime}$, and $\delta_{k k^{\prime}}=0$ otherwise). Provided that

$$
\iint\left|\left(x_{1}, x_{2}\right)\right|^{2} \mathrm{~d} x_{1} \mathrm{~d} x_{2}<\infty,
$$

it can be shown that [33]

$$
J\left(x_{1}, x_{2}\right)=\sum_{k} \lambda_{k} u_{k}\left(x_{1}\right) u_{k}^{*}\left(x_{2}\right),
$$

where $\lambda_{k}$ and $u_{k}(x)$ come from the eigenvalue equation given by Eq. (3). A similar expression holds for $K\left(x_{1}, x_{2}\right)$. A sufficient condition for Eq. (6) to hold is that the field is of finite energy; that is, the integral $\int J(x, x) \mathrm{d} x$ is finite. In the non-paraxial theory described in [31], this condition is always satisfied.

Eq. (7) is the spectral expansion of $J\left(x_{1}, x_{2}\right)$. It is also referred to as an outer-product expansion since its terms are outer products. In optics it is referred to as a coherent-mode representation [34,35], a terminology that will make sense in Section 4. This expression is also related to the process of diagonalizing a Hermitian-symmetric function, with $\lambda_{k} \delta_{k k^{\prime}}$ representing the diagonalized form of $J\left(x_{1}, x_{2}\right)$.

While establishing Eq. (7) in a rigorous manner is beyond the scope of this work, the reader may easily demonstrate its plausibility by substituting it in Eq. (3) to see that it indeed satisfies the eigenvalue equation. For a proper development see [33].

If Eq. (6) fails to hold, then it is possible that $J\left(x_{1}, x_{2}\right)$ has uncountably many orthonormal eigenfunctions, and possesses the expansion

$$
J\left(x_{1}, x_{2}\right)=\int \lambda(\nu) u_{\nu}\left(x_{1}\right) u_{\nu}^{*}\left(x_{2}\right) \mathrm{d} \nu .
$$

Stationary optical fields (Section 4.C) are an example for Eq. (8). When the field is stationary, all the complex exponentials $u_{\nu}(x)=e^{i 2 \pi \nu x}$ are an eigenfunction for $J\left(x_{1}, x_{2}\right)=$ $J\left(x_{1}-x_{2}\right)$, and we have

$$
J\left(x_{1}, x_{2}\right)=\int \lambda(\nu) e^{i 2 \pi \nu\left(x_{1}-x_{2}\right)} \mathrm{d} \nu,
$$

where $\lambda(\nu)$ is the Fourier transform of $f(x)=J(x, 0)$. It is easy to see that Eq. (9) is a particular case of Eq. (8). Also note that such cases where the energy may not be finite do not correspond to physical fields but rather idealizations or limiting cases of physical fields, much like sine-cosine or delta functions.

5. If $J\left(x_{1}, x_{2}\right)$ or $K\left(x_{1}, x_{2}\right)$ is expressible as the product of two functions $u^{\prime}\left(x_{1}\right)$ and $u^{\prime \prime}\left(x_{2}\right)$ as $u^{\prime}\left(x_{1}\right) u^{\prime \prime *}\left(x_{2}\right)$, then it follows from Hermitian symmetry that $u^{\prime}\left(x_{1}\right)$ and $u^{\prime \prime}\left(x_{2}\right)$ are related by a scalar factor, and further that it is possible to apply suitable scaling to express $J\left(x_{1}, x_{2}\right)$ or $K\left(x_{1}, x_{2}\right)$ in self-product form $u\left(x_{1}\right) u^{*}\left(x_{2}\right)$. That is, if we have a Hermitian-symmetric function that we can write in product form, it is possible to write it in self-product form. Being able to be written in product form is known as separability.

6. The statements that follow are equivalent: (i) $J\left(x_{1}, x_{2}\right)$ can be written in self-product form (or as a consequence of the previous item, just in product form). (ii) The eigenvalue sequence $\lambda_{k}$ is impulsive (non-zero for only one value of $k$ ); that is, $\lambda_{k}=\lambda_{0} \delta_{k k_{0}}$ for some $k_{0}$. See Appendix A. A similar equivalence holds for $K\left(x_{1}, x_{2}\right)$.

Later we will observe that functions satisfying one of the equivalent conditions given in item 6 represent light that is fully coherent. However, first we defined two new functions in the next section before we discuss incoherence and coherence in Section 4.

Before we leave this section, we cite two more results from [33]. If Eq. (6) holds true, then

$$
\sum_{k} \lambda_{k}=\int J(x, x) \mathrm{d} x
$$

and

$$
\sum_{k} \lambda_{k}^{2}=\iint\left|J\left(x_{1}, x_{2}\right)\right|^{2} \mathrm{~d} x_{1} \mathrm{~d} x_{2} .
$$

Equations (10) and (11) are stated in Section 5.25, Exercise 11 and Section 6.11, Exercise 11 of [33], respectively. While we again relegate matters of rigor to [33], we offer the following plausibility arguments for the above results. Integrating Eq. (7) along the diagonal line $x_{1}=x_{2}$ and using Eq. (5) leads to Eq. (10). Multiplying $J\left(x_{1}, x_{2}\right)$ with its conjugate, double integrating over $x_{1}$ and $x_{2}$, and again using Eq. (5) leads to Eq. (11).

\section{NORMALIZED MUTUAL INTENSITY}

Sometimes, we do not have special interest in the actual intensity of the field, but merely in the correlation of points with each other. When this is the case, it is convenient to employ the normalized versions of $J\left(x_{1}, x_{2}\right)$ and $K\left(x_{1}, x_{2}\right)$ : 


$$
L\left(x_{1}, x_{2}\right)=\frac{\left\langle f\left(x_{1}\right) f^{*}\left(x_{2}\right)\right\rangle}{\sqrt{\left\langle\left|f\left(x_{1}\right)\right|^{2}\right\rangle\left\langle\left|f\left(x_{2}\right)\right|^{2}\right\rangle}}=\frac{J\left(x_{1}, x_{2}\right)}{\sqrt{J\left(x_{1}, x_{1}\right) J\left(x_{2}, x_{2}\right)}}
$$

and

$$
\begin{aligned}
M\left(x_{1}, x_{2}\right) & =\frac{\left\langle\left(f\left(x_{1}\right)-\mu\left(x_{1}\right)\right)\left(f\left(x_{2}\right)-\mu\left(x_{2}\right)\right)^{*}\right\rangle}{\sqrt{\left\langle\left|f\left(x_{1}\right)-\mu\left(x_{1}\right)\right|^{2}\right\rangle\left\langle\left|f\left(x_{2}\right)-\mu\left(x_{2}\right)\right|^{2}\right\rangle}} \\
& =\frac{K\left(x_{1}, x_{2}\right)}{\sqrt{K\left(x_{1}, x_{1}\right) K\left(x_{2}, x_{2}\right)}} .
\end{aligned}
$$

The values of both of these functions are identically unity when $x_{1}=x_{2}$; that is, $L(x, x)=1$ and $M(x, x)=1$. Observe that $M\left(x_{1}, x_{2}\right)$ is just the normalized mutual intensity of the mean-subtracted field $f(x)-\mu(x)$. It can also be viewed as the correlation coefficient of the random variables $f\left(x_{1}\right)$ and $f\left(x_{2}\right)$ [32].

$L\left(x_{1}, x_{2}\right)$ and $M\left(x_{1}, x_{2}\right)$ exhibit similar properties to $J\left(x_{1}, x_{2}\right)$ and $K\left(x_{1}, x_{2}\right)$ in these ways:

1. Hermitian symmetry: $L\left(x_{1}, x_{2}\right)=L^{*}\left(x_{2}, x_{1}\right)$ and $M\left(x_{1}, x_{2}\right)=M^{*}\left(x_{2}, x_{1}\right)$.

2. $L\left(x_{1}, x_{2}\right)$ and $M\left(x_{1}, x_{2}\right)$ have real eigenvalues.

3. $L\left(x_{1}, x_{2}\right)$ and $M\left(x_{1}, x_{2}\right)$ are positive semi-definite with non-negative eigenvalues. Moreover, $\left|L\left(x_{1}, x_{2}\right)\right| \leq 1$ and $\left|M\left(x_{1}, x_{2}\right)\right| \leq 1$.

4. One can choose the eigenfunctions such that they are orthonormal, and similar to Eq. (7), in terms of their eigenfunctions and eigenvalues, $L\left(x_{1}, x_{2}\right)$ (or $M\left(x_{1}, x_{2}\right)$ ) can be decomposed as

$$
L\left(x_{1}, x_{2}\right)=\sum_{k} \lambda_{k} u_{k}\left(x_{1}\right) u_{k}^{*}\left(x_{2}\right),
$$

provided that

$$
\iint\left|L\left(x_{1}, x_{2}\right)\right|^{2} \mathrm{~d} x_{1} \mathrm{~d} x_{2}<\infty,
$$

similar to Eq. (6). If Eq. (15) does not hold, it may be the case that there exists uncountably many orthonormal eigenfunctions, and $L\left(x_{1}, x_{2}\right)$ (or $\left.M\left(x_{1}, x_{2}\right)\right)$ has the expansion

$$
L\left(x_{1}, x_{2}\right)=\int \lambda(\nu) u_{\nu}\left(x_{1}\right) u_{\nu}^{*}\left(x_{2}\right) \mathrm{d} \nu .
$$

5. If it is possible to write $L\left(x_{1}, x_{2}\right)$ or $M\left(x_{1}, x_{2}\right)$ in product form, it is also possible to write it in self-product form.

These additional properties also hold true:

1. The diagonal values of $L\left(x_{1}, x_{2}\right)$ and $M\left(x_{1}, x_{2}\right)$ for $x_{1}=x_{2}$ are equal to 1 . This is necessarily true since any point is by definition correlated with itself.

2. The following are equivalent (see Appendix A): (i) $L\left(x_{1}, x_{2}\right)$ (or $M\left(x_{1}, x_{2}\right)$ ) is separable (expressible in product form). (ii) The eigenvalue sequence of $L\left(x_{1}, x_{2}\right)$ (or $M\left(x_{1}, x_{2}\right)$ ) is impulsive (i.e., $\lambda_{k}=\lambda_{0} \delta_{k k_{0}}$ for some $k_{0}$ ). (iii) The function $L\left(x_{1}, x_{2}\right)$ (or $M\left(x_{1}, x_{2}\right)$ ) has unit magnitude everywhere: $\left|L\left(x_{1}, x_{2}\right)\right|=1$ (or $\left.\left|M\left(x_{1}, x_{2}\right)\right|=1\right)$.

Before we leave this section, here is the normalized mutual intensity counterpart of Eqs. (10) and (11):

$$
\sum_{k} \lambda_{k}=\int L(x, x) \mathrm{d} x=\int \mathrm{d} x
$$

and

$$
\sum_{k} \lambda_{k}^{2}=\iint\left|L\left(x_{1}, x_{2}\right)\right|^{2} \mathrm{~d} x_{1} \mathrm{~d} x_{2} .
$$

It follows as a corollary from Eq. (17) that if the optical field of interest extends from $-\infty$ to $+\infty$, then the sum of eigenvalues diverges.

\section{FULL INCOHERENCE AND FULL COHERENCE}

Partial coherence is a continuum with one end being full incoherence and the other end being full coherence. The basic concepts of partial coherence are covered in excellent textbooks such as $[3,36]$. Here we express full spatial incoherence and full spatial coherence mathematically in terms of the second-order correlation functions introduced above.

The basis of incoherence or coherence is statistical correlation. If any two arbitrary samples are completely correlated, we say the field is coherent. On the other hand, if any two arbitrary samples are completely uncorrelated, we say the field is incoherent. We will say that two random variables are completely correlated if their correlation with each other is as strong as it is with themselves. This finds mathematical expression in the magnitude of the normalized covariance (or correlation) being equal to unity. We will say that two random variables are completely uncorrelated if their normalized covariance (or correlation) is equal to zero. While complete incoherence and complete coherence are mathematical idealizations, a field can be effectively incoherent or coherent, if under the circumstances it effectively behaves as if it was completely incoherent or coherent (e.g., based on finite resolution or apertures).

Employing the normalized functions $L\left(x_{1}, x_{2}\right)$ and $M\left(x_{1}, x_{2}\right)$ has certain advantages. On the other hand, choosing between $L\left(x_{1}, x_{2}\right)$ and its mean-subtracted version $M\left(x_{1}, x_{2}\right)$ depends on how we prefer to define incoherence and coherence. This choice is discussed with care in the context of Young's experiment in [24]. In this present paper, we continued with the mutual intensity $J\left(x_{1}, x_{2}\right)$ and the normalized $L\left(x_{1}, x_{2}\right)$. The results for $K\left(x_{1}, x_{2}\right)$ and $M\left(x_{1}, x_{2}\right)$ are similar. While the mean-subtracted versions result in different definitions of incoherence and coherence, this is a non-essential difference.

The rationale for using $L$ may be alternatively explained as follows. The function $J$ reflects two different qualities of the field: (i) the spatial correlation (how correlated two points are with each other), and (ii) the spatial intensity distribution (how the intensity varies from one point to another). Now consider a $J$ function that is very concentrated along its diagonal. That means that distinct points have very low correlation and this is a field which is clearly on the incoherent side. However, also assume that the intensity is strong only in a certain location, but is very small elsewhere. Recalling that the eigenvalues of a diagonal matrix are merely its diagonal elements, it is not difficult to realize that this $J$ function will have a few large eigenvalues and many small eigenvalues.

In other words, although it is clearly a physically incoherent field, its eigenvalue distribution will not be uniform, but rather quite concentrated. This is because the effects of the intensity distribution on the eigenvalues interacts and is entangled with 
the effects of the correlation properties on the eigenvalues. In other words, $J$ contains information that situates the field both on the degree of coherence continuum, and on the degree of stationarity continuum. When we normalize $J$ to obtain $L$, we flatten the intensity along the diagonal and eliminate the effect of intensity on the eigenvalue distribution.

Now, the eigenvalue distribution reflects only the correlation properties. In other words, we have untangled the coherence continuum from the stationarity continuum. However, there is a side effect. No finite-energy physical signal can be stationary, but when we go from $J$ to $L$, the normalization introduces a partial stationarity ( $L$ does not strictly satisfy the definition of stationarity but nevertheless the uniformity imposed along the diagonal makes it closer to being stationary). This sometimes has the consequence of leading to a continuous eigenvalue spectrum for $L$.

\section{A. Full Incoherence}

Any two different samples of an incoherent field are uncorrelated, so that the mutual intensity function $J\left(x_{1}, x_{2}\right)$ and its normalized version $L\left(x_{1}, x_{2}\right)$ must be zero with the exception of the diagonal $x_{1}=x_{2}$. Furthermore, since $L(x, x)=1$ by definition, a fully incoherent continuous optical field has a normalized mutual intensity function of the following form,

$$
L\left(x_{1}, x_{2}\right)=\left\{\begin{array}{ll}
1 & \text { if } x_{1}=x_{2} \\
0 & \text { if } x_{1} \neq x_{2}
\end{array},\right.
$$

or alternatively if the associated mutual intensity function $J\left(x_{1}, x_{2}\right)$ is zero except when $x_{1}=x_{2}$.

It will be helpful to express Eq. (19) as a limit of the form

$$
L\left(x_{1}, x_{2}\right)=\lim _{W \rightarrow \infty} \operatorname{sinc}\left(W\left(x_{1}-x_{2}\right)\right) .
$$

For a fixed $W>0$, the eigenvalue equation for $L_{W}\left(x_{1}, x_{2}\right)=\operatorname{sinc}\left(W\left(x_{1}-x_{2}\right)\right)$ is

$$
\int \operatorname{sinc}\left(W\left(x-x^{\prime}\right)\right) u_{k}\left(x^{\prime}\right) \mathrm{d} x^{\prime}=\lambda_{k} u_{k}(x) .
$$

The left-hand side of Eq. (21) is the convolution of the sinc function with $u_{k}(x)$. This equation can be expressed in the Fourier domain as

$$
\frac{1}{W} \operatorname{rect}\left(\frac{\nu}{W}\right) \tilde{u}_{k}(\nu)=\lambda_{k} \tilde{u}_{k}(\nu),
$$

where $\tilde{u}_{k}(\nu)$ is the Fourier transform of $u_{k}(x)$. From Eq. (22), we can conclude that $\lambda_{k}=1 / W$ provided $\tilde{u}_{k}(\nu)$ is confined to the interval $[-W / 2, W / 2]$. We see that $L\left(x_{1}, x_{2}\right)$ is the limit of a family of functions $L_{W}\left(x_{1}, x_{2}\right)$ with all eigenvalues identical and equal to $1 / W$. Now, considering the limit $W \rightarrow \infty$, we see that the eigenvalue mass of $L\left(x_{1}, x_{2}\right)$ is spread as thinly and uniformly as is possible over all the values of $k$. Of course, this idealization is unphysical in the same sense that perfect sinusoids and delta functions are unphysical. Nevertheless, it serves as a useful formal device representing the limiting case of full incoherence.

As noted before, incoherence can be defined either in terms of $L\left(x_{1}, x_{2}\right)$ or $M\left(x_{1}, x_{2}\right)$. These definitions become identical when $f(x)$ has a zero mean. If samples of $f(x)$ are independent, $M\left(x_{1}, x_{2}\right)$ will equal 0 except when $x_{1}=x_{2}$, but $L\left(x_{1}, x_{2}\right)$ will not satisfy a similar property. When the samples are uncorrelated, $M\left(x_{1}, x_{2}\right)$ will equal to Eq. (19). In particular, this will be the case when the samples are identically distributed and independent, in which case the field will also be stationary.

\section{B. Full Coherence}

Any two samples of a coherent field are completely correlated, implying that the magnitude of the normalized correlation is equal to unity everywhere. Therefore the normalized mutual intensity $L\left(x_{1}, x_{2}\right)$ must have a magnitude of unity:

A fully coherent continuous optical field has a normalized mutual intensity function of

$$
\left|L\left(x_{1}, x_{2}\right)\right|=1 .
$$

All of the following conditions are equivalent to each other and to the above definition: (i) $J\left(x_{1}, x_{2}\right)$ is in self-product (or product) form. (ii) $L\left(x_{1}, x_{2}\right)$ is in self-product (or product) form. (iii) The eigenvalue function $\lambda_{k}$ of $J\left(x_{1}, x_{2}\right)$ is impulsive: $\lambda_{k}=\lambda_{0} \delta_{k k_{0}}$. (iv) The eigenvalue function $\lambda_{k}$ of $L\left(x_{1}, x_{2}\right)$ is impulsive: $\lambda_{k}=\lambda_{0} \delta_{k k_{0}}$.

The equivalence of these conditions are discussed in Appendix A. The eigenvalue function being impulsive means that there is only one non-zero eigenvalue. Any of these equivalent conditions can be used to define full coherence. The eigenvalue mass is concentrated at a single point as much as is possible. The same comments regarding the unphysicality of such idealizations as we have made in the incoherent case are applicable here as well. In reality, all systems have a finite spatial extent as well as a finite resolution so fully incoherent and fully coherent fields as defined do not exist.

Expressing the product form of $L\left(x_{1}, x_{2}\right)$ as $L\left(x_{1}, x_{2}\right)=u\left(x_{1}\right) u^{*}\left(x_{2}\right)$, we see that $u(x)$ must be of unit magnitude. In other words, the normalized correlation can be looked upon as the unnormalized correlation of another field that has been normalized to have unit magnitude. We also note that $u(x)$ is an eigenfunction of $L\left(x_{1}, x_{2}\right)$. This means that when we normalize $J\left(x_{1}, x_{2}\right)$ to obtain $L\left(x_{1}, x_{2}\right)$, what we have effectively done is to remove the effect of varying spatial intensity from the field. We would want to do this when we are less interested in the varying spatial intensity and more interested in the relative correlation between points.

One class of fields that are coherent are deterministic fields. For such fields, the ensemble average is not necessary, and the function $J\left(x_{1}, x_{2}\right)$ is already in product form.

\section{Stationarity}

Here we briefly touch upon the concept of stationarity, in particular, second-order stationarity.

Stationary optical fields have mutual intensity functions that are shift-invariant:

$$
J\left(x_{1}, x_{2}\right)=J\left(x_{1}-x_{2}\right) .
$$

This means that the second-order correlation depends only on the distance between $x_{1}$ and $x_{2}$ and not on their absolute values: If $J\left(x_{1}, x_{2}\right)$ is spatially stationary, so is $L\left(x_{1}, x_{2}\right)$.

\section{MEASURES OF THE PARTIAL COHERENCE OF A FIELD}

In the previous section we established the two limiting end points of partial coherence, full incoherence and full coherence, 
and presented definitions. We now propose five scalar measures for the degree of partial coherence in terms of these correlation functions. This can be achieved by interpolating some particular feature of the functions. For instance, we observed the eigenvalue mass of $L\left(x_{1}, x_{2}\right)$ for incoherent fields to be uniformly spread, and the eigenvalue mass for a coherent field to be concentrated at a single point. Thus an appropriate function of the eigenvalues that interpolates between these two extremes is a candidate for a such a measure. On the other hand, the function $L\left(x_{1}, x_{2}\right)$ corresponding to an incoherent field is zero except when $x_{1}=x_{2}$, representing maximum concentration, while the function $L\left(x_{1}, x_{2}\right)$ corresponding to a coherent field is everywhere equal to unity, representing maximum spread. Again, an appropriate interpolation is a candidate for a such a measure.

There are multiple ways to create such an interpolation, resulting in multiple candidate definitions. We propose selected candidate definitions. The definitions we have presented are not exhaustive, but illustrate the different possibilities. The numbering of the different definitions presented are in oneto-one correspondence with the numbering used in [24], where corresponding definitions were offered for fields of discrete variables.

In Subsection 4.A, we will consider eigenvalue-distributionbased measures. Eigenvalue distributions are useful for various problems of statistical optics, such as the theory of laser resonator modes, and some propagation problems with partially coherent light ([4], Chapter 5). They can be interpreted as the strength of various coherent modes. In Subsection 4.B, we considered mutual intensity function based measures.

The mutual intensity function manifests itself in a variety of experiments and applications. Perhaps the best known experiment illustrating the physical meaning of mutual intensity is Young's double-pinhole experiment. Here, the intensity at an observation point depends on the value of the mutual intensity function at the pinholes. Stellar interferometry, propagation through free space, transmission through optical systems, image formation, the Van Cittern-Zernike theorem, and scattering from deterministic or random media are some applications illustrating the usefulness of mutual intensity functions.

We defined our measures $c^{\prime}$ such that at first the smallest value $c_{\min }^{\prime}$ corresponds to full incoherence or coherence, and the largest value $c_{\max }^{\prime}$ corresponds to full coherence or incoherence. We then employed an appropriate map to arrive at the final measure denoted by $c$, which takes the value 0 for incoherent light and takes the value 1 for coherent light.

To ensure that $c$ stays within the interval $[0,1]$ and due to the smooth transition it affords, we found an arctangent map of the form $c=\frac{2}{\pi} \arctan \left(c^{\prime} / \alpha\right)$ to be useful. By adjusting the value of $\alpha$, one can obtain a smoother or more abrupt dependence. In what follows we will take $\alpha=1$. Other functions, such as $c=\left(1+\alpha / c^{\prime}\right)^{-1}$ could also be used, but the specific type of function employed usually does not have a substantial effect.

\section{A. Eigenvalue-Distribution-Based Measures}

Here we considered the eigenvalue sequence $\lambda_{k}$ of the function $L\left(x_{1}, x_{2}\right)$ as the basis for defining a scalar measure. We have seen that light that is nearly incoherent is characterized by very small eigenvalues with the eigenvalue mass being uniformly spread over $k$. In contrast, we saw that coherent light is characterized by the distribution $\lambda_{k}=\lambda_{0} \delta_{k k_{0}}$ with the eigenvalue mass concentrated at a point. The incoherent limit is characterized by maximal uniform spread of the eigenvalue mass, whereas the coherent limit is characterized by maximal concentration of the eigenvalue mass.

To provide some motivation for our definitions, consider the eigenvalue distribution normalized to yield a sequence that adds up to unity: $\lambda_{1} / \sum_{k=1}^{N} \lambda_{k}, \ldots, \lambda_{N} / \sum_{k=1}^{N} \lambda_{k}$. This makes it possible to view this distribution as if it were a discrete probability distribution. Thus the reader will observe upon reading further that Definition 1 can be interpreted as the second moment of a probability distribution and Definition 3 can be interpreted as its entropy. In Definition 1, the order in which the $\lambda_{k}$ are indexed with respect to $k$ makes a big difference. Shuffling the positions of the $\lambda_{k}$ can change the second moment considerably. The second moment is a measure of the spread of the distribution and thus tells us whether the distribution is more concentrated or more uniformly spread.

In Definition 3, on the other hand, the order in which the $\lambda_{k}$ are indexed with respect to $k$ makes no difference. The entropy is not a measure of functional spread but a measure of uniformity among a group of numbers. It again tells us whether the distribution is concentrated or not, but in a different way. Finally, Definition 2 does not involve the probability distribution interpretation. It is inspired by the fact that the sum of the squares of a non-negative sequence is always less than or equal to the square of the sum, with equality when only one member of the sequence is non-zero. Thus the ratio of the sum of the squares, to the square of the sum, will inform us whether the eigenvalues are concentrated or not.

As explained in Section 3, $L\left(x_{1}, x_{2}\right)$ may have uncountably many eigenfunctions and thus possess a continuous eigenvalue spectrum if Eq. (15) is not satisfied. In the definitions discussed below, we address this case as well. However, the continuous spectrum counterpart of Definition 1 in [24] does not constitute a very useful measure and is discussed only for completeness.

Definition 1: Taking Definition 1 in [24] into account, we propose our first measure as

$$
c_{1}^{\prime}=\lim _{N \rightarrow \infty} \sum_{n=1}^{N}(n-1)^{2} \frac{\lambda_{n}}{\sum_{k=1}^{N} \lambda_{k}} .
$$

In Eq. (25), we assumed that the eigenvalues are ordered (i.e., $\lambda_{1} \geq \lambda_{2} \geq \ldots \geq \lambda_{n} \geq \ldots$ ). For coherent light, $\lambda_{n}$ is nonzero only for $n=1$ so that $c_{1}^{\prime}=0$. For incoherent light, we have

$$
c_{1}^{\prime}=\lim _{N \rightarrow \infty} \frac{1}{N} \sum_{n=1}^{N}(n-1)^{2}=\infty .
$$

Mapping this to between 0 and 1 with an inverse tangent function of its inverse, we finally obtained

$$
\begin{aligned}
c_{1} & =\frac{2}{\pi} \arctan \left(\frac{1}{c_{1}^{\prime}}\right) \\
& =\lim _{N \rightarrow \infty} \frac{2}{\pi} \arctan \left[\sum_{n=1}^{N}(n-1)^{2} \frac{\lambda_{n}}{\sum_{n=1}^{N} \lambda_{n}}\right]^{-1},
\end{aligned}
$$


from which we obtained $c_{1}=0$ for incoherent light, and $c_{1}=1$ for coherent light. This definition exhibits the defect that, certain slowly decaying eigenvalue sequences may also have $c_{1}=0$, despite the fact that they do not strictly satisfy our definition of full incoherence. It does not seem this defect would have much effect in practice, since such distributions would usually correspond to effectively incoherent fields.

If $L\left(x_{1}, x_{2}\right)$ does not satisfy Eq. (15), then it is likely that it has an expansion given by Eq. (16). If this is the case, based on the behavior of the function $\lambda(\nu)$ for the coherent and incoherent limits, we might say that the more the concentration of the eigenvalue distribution, the more the coherence of the light. On the other hand, the more uniformly spread the distribution, the more incoherent the light. Since the variance is a common measure of the spread and concentration of a function, we might consider the following measure,

$$
c_{1}^{\prime}=\int(\nu-\bar{\nu})^{2} \lambda(\nu) \mathrm{d} \nu,
$$

where $\bar{\nu}=\int \nu \lambda(\nu) \mathrm{d} \nu$. This measure becomes zero for the coherent limit and tends to infinity for the incoherent limit. However, that this measure is less than satisfactory becomes evident when we consider certain special cases. For instance, consider the eigenvalue distribution $\lambda(\nu)=A \operatorname{rect}\left(\frac{\nu-\nu_{0}}{\Delta \nu}\right)+$ $A \operatorname{rect}\left(\frac{\nu+\nu_{0}}{\Delta \nu}\right)$, where $\nu_{0}$ and $A$ have relatively large values and $\Delta \nu$ is very small. In this case $c_{1}^{\prime}$ will have a large value, but the eigenvalue distribution is nowhere near the uniform distribution we associate with the incoherent limit. The variance measures distribution of the mass of a function from its center of gravity, but does not measure how uniformly this mass is distributed. For this reason, the variance is a more meaningful measure of concentration at a point versus uniform spread, for functions which monotonically decrease away from the center of gravity. In the discrete counterpart of this definition [24], we ensured monotonicity by assuming that the eigenvalues are indexed in decreasing order, a common practice with discrete eigenvalue or singular-value distributions [37]. While the same should in principle be possible for continuous eigenvalue spectrums, this is neither a convenient nor common practice. Therefore, we do not offer the variance of the eigenvalue distribution as a measure of partial coherence for fields of continuous variables.

Definition 2: The second measure we considered is

$$
c_{2}=\lim _{N \rightarrow \infty} \frac{\sum_{n=1}^{N} \lambda_{n}^{2}}{\left(\sum_{n=1}^{N} \lambda_{n}\right)^{2}} .
$$

For light that is very nearly incoherent, the eigenvalues are very small and uniformly distributed, which implies

$$
c_{2}=\lim _{N \rightarrow \infty} \frac{1}{N}=0 .
$$

Moreover, for the coherent light, we get $c_{2}=\lambda_{0}^{2} / \lambda_{0}^{2}=1$. So, the measure of coherence proposed by Eq. (29) works in the two extreme cases as desired.

A similar measure has received attention in a number of earlier works [38-45]. However, we should note that our definition is based on the normalized mutual intensity rather than its unnormalized version. This will be further discussed in Definition 3.
On the other hand, if $L\left(x_{1}, x_{2}\right)$ does not satisfy Eq. (15) and has a continuous eigenfunction expansion given by Eq. (16), we argue that the measure considered in Definition 1 is based on thinking of the distribution of eigenvalues as a function of the index $\nu$, with the variance of this distribution essentially corresponding to a spread over $\nu$. But the distribution of eigenvalues can be alternatively measured without referring to any indexing variable, but just as the internal spread among a collection of numbers. So we chose the variance of the eigenvalues instead of the variance of the eigenvalue distribution function,

$$
c_{2}^{\prime}=\int[\lambda(\nu)-\bar{\lambda}]^{2} \mathrm{~d} \nu=\int \lambda^{2}(\nu) \mathrm{d} \nu,
$$

where $\bar{\lambda}=\int \lambda(\nu) \mathrm{d} \nu / \int \mathrm{d} \nu=0$ is the mean value of the eigenvalues. The integral $\int \lambda^{2}(\nu) \mathrm{d} \nu$ evaluates to 0 for the incoherent limit. As we near the limit of full coherence, the value of this integral will approach $\infty$. To map $\int \lambda^{2}(\nu) \mathrm{d} \nu$ to the interval $[0$, $1]$, we chose the arctangent function to obtain

$$
c_{2}=\frac{2}{\pi} \arctan \left[\int \lambda^{2}(\nu) \mathrm{d} \nu\right] .
$$

For the incoherent case $c_{2}=0$ and for the coherent case $c_{2}=1$, as desired. Intermediate values will be obtained for general partially coherent fields. When $\lambda(\nu)$ is such that $\int \lambda^{2}(\nu) \mathrm{d} \nu=1$, then $c_{2}=0.5$. We may consider this case to be the geometric midway between full incoherence and full coherence.

Definition 3: To define our third measure, similar to the previous measures, we assumed the eigenvalues are arranged in descending order (i.e., $\lambda_{1} \geq \lambda_{2} \geq \ldots \geq \lambda_{n} \geq \ldots$ ). Then, as our measure of coherence, we considered the entropy of the random variable having the following discrete probability mass function,

$$
\left(\frac{\lambda_{1}}{\sum_{n=1}^{N} \lambda_{n}}, \frac{\lambda_{2}}{\sum_{n=1}^{N} \lambda_{n}}, \ldots, \frac{\lambda_{N}}{\sum_{n=1}^{N} \lambda_{n}}\right),
$$

where we let $N$ go to infinity. In other words, we normalized the eigenvalue distribution so that it exhibits the properties of a probability distribution. Then we inquired whether the concept of entropy, measuring disorder versus maximum order, might form the basis of a suitable measure. A particular realization of a nearly incoherent process provides a significant amount of information, whereas a particular realization of a nearly coherent process provides a minimal amount of information. So we defined our measure to be the entropy of the random variable defined by this probability distribution:

$$
c_{3}^{\prime}=-\lim _{N \rightarrow \infty} \sum_{n=1}^{N} \frac{\lambda_{n}}{\sum_{k=1}^{N} \lambda_{k}} \log \frac{\lambda_{n}}{\sum_{k=1}^{N} \lambda_{k}} .
$$

For coherent light, we can see that $c_{3}^{\prime}=0$. For incoherent light, we have $c_{3}^{\prime}=\lim _{N \rightarrow \infty} \log N=\infty$. Therefore, we define $c_{3}$ as

$$
c_{3}=-\lim _{N \rightarrow \infty} \frac{2}{\pi} \arctan \left(\sum_{n=1}^{N} \frac{\lambda_{n}}{\sum_{k=1}^{N} \lambda_{k}} \log \frac{\lambda_{n}}{\sum_{k=1}^{N} \lambda_{k}}\right)^{-1},
$$

which assumes the values $c_{3}=0$ for incoherent light and $c_{3}=1$ coherent light. As we have noted in [24], Gamo seems 
to have been the first to propose a definition of the degree of partial coherence based on entropy [46,47]. The entropy concept is also used similarly in $[48,49]$. The definition in $[46,47]$ was based on the eigenvalue distribution of the mutual intensity without normalization. However, if we do not perform normalization, the incoherent limit cannot be appropriately characterized. The approach taken in [24], which relies on normalized matrices, and the approach taken in this paper, which relied on normalized functions, solved these issues.

On the other hand, if Eq. (15) is not satisfied by $L\left(x_{1}, x_{2}\right)$, and $L\left(x_{1}, x_{2}\right)$ has an expansion given by Eq. (16) instead of Eq. (14), we must proceed as follows: Note that the eigenvalue mass distribution function $p(\nu)=\frac{\lambda(\nu)}{\int \lambda\left(\nu^{\prime}\right) \mathrm{d} \nu^{\prime}}$ exhibits the properties of a probability density function. If $p(\nu) \geq 0$ is a probability density function satisfying $\int p(\nu) \mathrm{d} \nu=1$, the entropy is defined as $-\int p(\nu) \log p(\nu) \mathrm{d} \nu$. (In continuous contexts, the term differential entropy is also employed.) Since the distribution of the eigenvalue mass $p(\nu)$ satisfies the same basic properties as a probability density function, the following definition naturally asserts itself:

$$
\begin{aligned}
c_{3}^{\prime} & =-\int p(\nu) \log p(\nu) \mathrm{d} \nu \\
& =-\int \frac{\lambda(\nu)}{\int \lambda\left(\nu^{\prime}\right) \mathrm{d} \nu^{\prime}} \log \left(\frac{\lambda(\nu)}{\int \lambda\left(\nu^{\prime}\right) \mathrm{d} \nu^{\prime}}\right) \mathrm{d} \nu .
\end{aligned}
$$

The base of the logarithm does not matter but may be chosen as $e$ for concreteness. In the incoherent limit we have $c_{3}^{\prime}=\infty$ and in the coherent limit we have $c_{3}^{\prime}=-\infty$. Therefore, we define

$$
c_{3}=\frac{1}{\pi} \arctan \left[\int \frac{\lambda(\nu)}{\int \lambda\left(\nu^{\prime}\right) \mathrm{d} \nu^{\prime}} \log \left(\frac{\lambda(\nu)}{\int \lambda\left(\nu^{\prime}\right) \mathrm{d} \nu^{\prime}}\right) \mathrm{d} \nu\right]+\frac{1}{2} .
$$

For very nearly incoherent fields for which the eigenvalues are very small and uniformly distributed we find $c_{3}=0$ and for the coherent limit for which $\lambda(\nu)=\lambda_{0} \delta\left(\nu-\nu_{0}\right)$ we find $c_{3}=1$.

It may be interesting to explore the class of functions-and the optical fields they represent-that satisfy $\int \lambda^{2}(\nu) \mathrm{d} \nu=1$ or $\int \lambda(\nu) \log \lambda(\nu) \mathrm{d} \nu=0$ subject to $\int \lambda(\nu) \mathrm{d} \nu=1$ and $\lambda(\nu) \geq 0$. Such fields correspond to the geometric midway between fully incoherent and fully coherent light fields, with respect to Definitions 2 and 3, respectively. An example of an eigenvalue distribution satisfying both conditions is $\lambda(\nu)=\operatorname{rect}(\nu)$, a function which is intuitively acceptable as the geometric midway between maximal uniform spread and maximal concentration at a point. An example of a normalized mutual intensity function having such an eigenvalue distribution is $L\left(x_{1}, x_{2}\right)=\operatorname{sinc}\left(x_{1}-x_{2}\right)$. In fact, radiation from an incoherent source having intensity $I(x)=\operatorname{rect}(x / \ell)$ in free space results in a normalized intensity function $L\left(x_{1}, x_{2}\right)=\operatorname{sinc} \frac{\ell\left(x_{1}-x_{2}\right)}{\lambda d}$ ([36], Chapter 11.3), where $d$ is the distance between the source and observation planes, constituting a physical example for a continuous spectrum of rectangular form.

Before closing this section, we briefly discuss how the different measures considered can be compared. One might wonder whether the measures considered use the range $[0,1]$ in the same way. Ideally, one would expect that the fields that one measure maps, for example, close to 0 , should again be mapped close to 0 by the other measures as well. To this end we will introduce additional parameters to the measures. For example, instead of $c_{1}^{\prime}$ as given by Eq. (25), one might consider

$$
c_{1}^{\prime}(\beta)=\lim _{N \rightarrow \infty} \sum_{n=1}^{N}(n-1)^{2}\left(\frac{\lambda_{n}}{\sum_{n=1}^{N} \lambda_{n}}\right)^{\beta},
$$

with $\beta \geq 1$. With this definition, it can be shown that $c_{1}^{\prime}(\beta)$ and $\left(c_{2}\right)^{3}$ asymptotically behave the same for the same decay rate of the sequence $\lambda_{n} / \sum_{k=1}^{N} \lambda_{k}$, when $\beta=6$. Alternatively, instead of the Shannon entropy giving rise to Eq. (33), one can make use of the Rényi entropy [50], and define

$$
c_{3}^{\prime}(\gamma)=\frac{1}{1-\gamma} \lim _{N \rightarrow \infty} \log \left[\sum_{n=1}^{N}\left(\frac{\lambda_{n}}{\sum_{k=1}^{N} \lambda_{k}}\right)^{\gamma}\right],
$$

with $\gamma \geq 1$. Comparing Eq. (29) with Eq. (38), we observed that Definition 2 is related to the Rényi entropy approach for $\gamma=2$ through $c_{3}^{\prime}(2)=-\log \left(c_{2}\right)$. Hence, we see that the three eigenvalue-based definitions are connected to each other.

\section{B. Function-Spread-Based Measures}

We now focus on the normalized mutual intensity function $L\left(x_{1}, x_{2}\right)$ itself, instead of its eigenvalues. When $L\left(x_{1}, x_{2}\right)$ is zero except when $x_{1}=x_{2}$, we have incoherent light. When $L\left(x_{1}, x_{2}\right)$ has unit magnitude everywhere, we have coherent light. By interpolating between these endpoints, we can define the measures of degree of partial coherence that follow.

Definition 4: We considered the moment of inertia (the spatial variance) around the line $x_{1}=x_{2}$ in the $x_{1}-x_{2}$ plane. Since $\left|L\left(x_{1}, x_{2}\right)\right|=\left|L\left(x_{2}, x_{1}\right)\right|$ from Hermitian symmetry, we proposed the measure of spread:

$$
c_{4}^{\prime}=\lim _{W \rightarrow \infty} \frac{\int_{-W / 2}^{W / 2} \int_{-W / 2}^{W / 2}\left(x_{1}-x_{2}\right)^{2}\left|L\left(x_{1}, x_{2}\right)\right|^{2} \mathrm{~d} x_{1} \mathrm{~d} x_{2}}{\int_{-W / 2}^{W / 2} \int_{-W / 2}^{W / 2}\left|L\left(x_{1}, x_{2}\right)\right|^{2} \mathrm{~d} x_{1} \mathrm{~d} x_{2}} .
$$

For incoherent light, we have $c_{4}^{\prime}=0$ and for coherent light for which $\left|L\left(x_{1}, x_{2}\right)\right|=1$ we have $c_{4}^{\prime}=\infty$. Defining

$$
c_{4}=\frac{2}{\pi} \arctan \left(c_{4}^{\prime}\right) \text {, }
$$

we obtain a measure that is 1 for the fully coherent case and 0 for the fully incoherent case. If the optical field of interest is defined only on a finite interval, then instead of using the arctan function, it is sufficient to divide $c_{4}^{\prime}$ by the maximum value it can attain so that $c_{4}$ is 1 for the coherent case.

However, a problem with the coherent limit becomes apparent upon closer inspection. Even when the field is not fully coherent and $\left|L\left(x_{1}, x_{2}\right)\right| \neq 1$, it is possible for $c_{4}^{\prime}=\infty$ and $c_{4}=1$, if the decay of $L\left(x_{1}, x_{2}\right)$ away from the line $x_{1}=$ $x_{2}$ is relatively slow. An alternative approach would be to replace the denominator of Eq. (39) with $\iint \mathrm{d} x_{1} \mathrm{~d} x_{2}$ instead, leading to a definition more analogous to the discrete counterpart of this definition given in [24]. Although in this case it is easy to see that the value of $c_{4}^{\prime}$ for the fully coherent case is always strictly greater than all other cases, it is still possible for some of these partially coherent cases to have $c_{4}^{\prime}=\infty$, so that this approach does not fully solve the problem either. 
Definition 5: Another option is to measure the energy of $L\left(x_{1}, x_{2}\right)$ without paying attention to its spatial distribution. Recalling that $L\left(x_{1}, x_{2}\right) \leq 1$ and that $L(x, x)=1$, this measure will be minimum when non-zero values of $L\left(x_{1}, x_{2}\right)$ are limited to the line $x_{1}=x_{2}$ (incoherent light) and maximum when the values of $L\left(x_{1}, x_{2}\right)$ are of unit magnitude (corresponding to coherent light):

$$
c_{5}^{\prime}=\iint\left|L\left(x_{1}, x_{2}\right)\right|^{2} \mathrm{~d} x_{1} \mathrm{~d} x_{2}
$$

A suitable normalization would be

$$
\begin{aligned}
c_{5} & =\lim _{W \rightarrow \infty} \frac{\int_{-W / 2}^{W / 2} \int_{-W / 2}^{W / 2}\left|L\left(x_{1}, x_{2}\right)\right|^{2} \mathrm{~d} x_{1} \mathrm{~d} x_{2}}{\left(\int_{-W / 2}^{W / 2} L(x, x) \mathrm{d} x\right)^{2}} \\
& =\lim _{W \rightarrow \infty} \frac{\int_{-W / 2}^{W / 2} \int_{-W / 2}^{W / 2}\left|L\left(x_{1}, x_{2}\right)\right|^{2} \mathrm{~d} x_{1} \mathrm{~d} x_{2}}{W^{2}} .
\end{aligned}
$$

Combining Eq. (42) with Eq. (17) and Eq. (18), we see that this definition of measure is not essentially different from Eq. (29) and Definition 2. Similarly to Definition 2, we have $c_{5}=0$ for the incoherent light and $c_{5}=1$ for the coherent light.

\section{FOURIER-DOMAIN ANALYSIS AND DUALITY}

In an earlier section, the definitions of incoherence and coherence were expressed in terms of autocorrelations of the field $f(x)$. Now we express equivalent conditions for incoherence and coherence in the spectral domain.

Denoting the Fourier transform of a function $f(x)$ by $\tilde{f}(\nu)=\int f(x) \exp (-i 2 \pi \nu x) \mathrm{d} x$, we could express the mutual intensity $J_{\tilde{f}}$ of the Fourier transform as

$$
\begin{aligned}
J_{\tilde{f}}\left(\nu_{1}, \nu_{2}\right)= & \left\langle\left(\int f\left(x_{1}\right) e^{-i 2 \pi \nu_{1} x_{1}} \mathrm{~d} x_{1}\right)\right. \\
& \left.\times\left(\int f\left(x_{2}\right) e^{-i 2 \pi \nu_{2} x_{2}} \mathrm{~d} x_{2}\right)^{*}\right\rangle \\
= & \iint e^{-i 2 \pi \nu_{1} x_{1}} J_{f}\left(x_{1}, x_{2}\right) e^{i 2 \pi \nu_{2} x_{2}} \mathrm{~d} x_{1} \mathrm{~d} x_{2} .
\end{aligned}
$$

We see that the mutual intensity of the Fourier transform can be expressed as the double Fourier transform of the mutual intensity of the original function.

A mutual intensity function $J_{f}\left(x_{1}, x_{2}\right)$ that can be expressed in the form $J_{f}\left(x_{1}-x_{2}\right)$ is a second-order stationary random process. (If the function $J_{f}\left(x_{1}-x_{2}\right)$ represented the integral kernel of a linear system, the functional dependence on $x_{1}$ $x_{2}$ would correspond to a space-invariant system.) The complex exponentials are known to be the eigenfunctions of kernel functions that can be expressed as functions of the difference $x_{1}-x_{2}$. Therefore the double Fourier transform will "diagonalize" such functions, in the sense of the following equation:

$$
\begin{aligned}
J_{\tilde{f}}\left(\nu_{1}, \nu_{2}\right) & =\iint e^{-i 2 \pi \nu_{1} x_{1}} J_{f}\left(x_{1}-x_{2}\right) e^{i 2 \pi \nu_{2} x_{2}} \mathrm{~d} x_{1} \mathrm{~d} x_{2} \\
& =\Lambda_{J}\left(\nu_{1}\right) \delta\left(\nu_{1}-\nu_{2}\right) .
\end{aligned}
$$

To put it differently, a second-order stationary field is represented by a function of $x_{1}-x_{2}$ in the spatial coordinate domain but by an impulsive function in the spectral domain.
Conversely, if $J_{f}\left(x_{1}, x_{2}\right)$ is impulsive (non-zero only when $\left.x_{1}=x_{2}\right)$, we have a field that is incoherent, and $\tilde{f}_{\tilde{f}}\left(\nu_{1}, \nu_{2}\right)$ will exhibit the form $J_{\tilde{f}}\left(\nu_{1}-\nu_{2}\right)$. That is, a field that is incoherent is represented by an impulsive function in the spatial coordinate domain but by a function of $\nu_{1}-\nu_{2}$ in the spectral domain. In other words, stationarity and incoherence are Fourier conjugates or Fourier duals.

If it is the case that $J_{f}\left(x_{1}, x_{2}\right)$ is expressible in product form (separability), then it is possible to show that $J_{\tilde{f}}\left(\nu_{1}, \nu_{2}\right)$ is also expressible in product form. To put it differently, coherent fields are represented by separable functions in both the spatial coordinate and spectral domains; coherence is self-dual.

\section{CONCLUSION}

We presented the continuous space-variable version of the linear algebraic theory of partial coherence presented for discrete variables in [24]. We believe this approach facilitates the use of linear algebraic techniques and makes it possible to precisely define the concepts of incoherence and coherence in a mathematical way. Such a formulation may be beneficial in optical signal processing where the light is of a partially coherent nature.

Several measures for the scalar degree of partial coherence of a field were proposed. These measures are equal to unity for full coherence and equal to zero for full incoherence. We first considered eigenvalue-distribution-based measures for which the coherent limit corresponds to only one non-zero eigenvalue and the incoherent limit corresponds to a uniform eigenvalue distribution. We saw that measures of the width of the eigenvalue distribution function are not always satisfactory in the incoherent limit, since increasing width does not always coincide with increasing uniformity. For this reason, measures such as Definitions 2 and 3, which are based on the distribution of eigenvalues without indexing them with respect to some variable, seem more attractive.

We can make a similar observation for function spreadbased measures for which the incoherent limit corresponds to a normalized mutual intensity that is non-zero only along the diagonal and the coherent limit corresponds to a normalized mutual intensity with unit magnitude everywhere. Since measures of width of the normalized mutual intensity do not always serve as satisfactory measures of uniformity in the coherent limit, measures such as Definition 5, which measure how close the normalized correlation values are to unity, are more attractive. We also saw that Definition 5 was essentially similar to Definition 2.

In [24] we discussed Young's experiment to relate the mathematical definitions to our physical understanding of coherence. We also discussed the use of correlation versus covariance functions. These discussions are directly applicable to the continuous formulation discussed in this paper and thus have not been repeated.

\section{APPENDIX A: ALTERNATIVE DEFINITIONS OF FULL COHERENCE}

Here we show that the following are equivalent, so that any one of them can be taken as the definition of full coherence: 
(i) $J\left(x_{1}, x_{2}\right)$ is in self-product (or product) form.

(ii) $L\left(x_{1}, x_{2}\right)$ is in self-product (or product) form.

(iii) For every $x_{1}$ and $x_{2},\left|L\left(x_{1}, x_{2}\right)\right|=1$.

(iv) Eigenvalue sequence $\lambda_{k}$ of $J\left(x_{1}, x_{2}\right)$ is impulsive: $\lambda_{k}=\lambda_{0} \delta_{k k_{0}}$. If Eq. (8) holds, then $\lambda(\nu)=\lambda_{0} \delta\left(\nu-\nu_{0}\right)$.

(v) Eigenvalue sequence $\lambda_{k}$ of $L\left(x_{1}, x_{2}\right)$ is impulsive: $\lambda_{k}=\lambda_{0} \delta_{k k_{0}}$. If Eq. (16) holds, then $\lambda(\nu)=\lambda_{0} \delta\left(\nu-\nu_{0}\right)$.

If $J\left(x_{1}, x_{2}\right)$ is replaced by $K\left(x_{1}, x_{2}\right)$ and $L\left(x_{1}, x_{2}\right)$ is replaced by $M\left(x_{1}, x_{2}\right)$, the above equivalences still hold, and exactly the same proofs below are applicable.

Proof of (i) $\Leftrightarrow$ (iv): That (iv) implies (i) is easily seen from the spectral expansion given in Eq. 8 for the continuous spectrum case and Eq. 7 for the discrete spectrum case. To show that (i) implies (iv), let $J\left(x_{1}, x_{2}\right)=u\left(x_{1}\right) u^{*}\left(x_{2}\right)$ for some $u(x)$. Substituting this in the eigenvalue equation (3) we obtain

$$
\int u\left(x_{1}\right) u^{*}\left(x_{2}\right) u_{k}\left(x_{2}\right) \mathrm{d} x_{2}=\lambda_{k} u_{k}\left(x_{1}\right)
$$

and

$$
u\left(x_{1}\right) \int u^{*}\left(x_{2}\right) u_{k}\left(x_{2}\right) \mathrm{d} x_{2}=\lambda_{k} u_{k}\left(x_{1}\right)
$$

for all $k$. If $\lambda_{k} \neq 0$ for some $k$, then $\int u^{*}\left(x_{2}\right) u_{k}\left(x_{2}\right) \mathrm{d} x_{2} \neq 0$ follows necessarily. This implies that $u\left(x_{1}\right) \propto u_{k}\left(x_{1}\right)$ for all $k$ for which $\lambda_{k} \neq 0$. Since $u\left(x_{1}\right)$ cannot be proportional to more than one member of an orthonormal set, it follows that $\lambda_{k} \neq 0$ is valid for only one value of $k$. Now let $k_{0}$ be the only number for which $\lambda_{k}$ is nonzero. Since $u\left(x_{1}\right) \propto u_{k_{0}}\left(x_{1}\right)$, we have $u\left(x_{1}\right)=A u_{k_{0}}\left(x_{1}\right)$ for some possibly complex number $A$. Then from Eq. (A2) we obtain

$$
A u_{k_{0}}\left(x_{1}\right) A^{*} \int u_{k_{0}}^{*}\left(x_{2}\right) u_{k_{0}}\left(x_{2}\right) \mathrm{d} x_{2}=\lambda_{k_{0}} u_{k_{0}}\left(x_{1}\right)
$$

and

$$
|A|^{2} u_{k_{0}}\left(x_{1}\right)=\lambda_{k_{0}} u_{k_{0}}\left(x_{1}\right) \text {. }
$$

Hence $\lambda_{k_{0}}=|A|^{2}$ and $\lambda_{k}=0$ otherwise. In other words, $\lambda_{k}=\lambda_{0} \delta_{k k_{0}}$, where $\lambda_{0}=|A|^{2}$. The proof for the case when Eq. (8) holds is similar.

Proof of (ii) $\Leftrightarrow$ (v): Follows from the proof of (i) $\Leftrightarrow$ (iv) in an identical manner.

Proof of (i) $\Rightarrow$ (ii): Given $J\left(x_{1}, x_{2}\right)=u\left(x_{1}\right) u^{*}\left(x_{2}\right)$ we can write

$$
\begin{aligned}
L\left(x_{1}, x_{2}\right) & =\frac{J\left(x_{1}, x_{2}\right)}{\sqrt{J\left(x_{1}, x_{1}\right) J\left(x_{2}, x_{2}\right)}} \\
& =\left(\frac{u\left(x_{1}\right)}{\sqrt{J\left(x_{1}, x_{1}\right)}}\right)\left(\frac{u^{*}\left(x_{2}\right)}{\sqrt{J\left(x_{2}, x_{2}\right)}}\right)
\end{aligned}
$$

so that $L\left(x_{1}, x_{2}\right)$ is also separable.

Proof of (ii) $\Rightarrow$ (iii): Since $|L(x, x)|=1$ holds for all $x$, we have $u(x) u^{*}(x)=|u(x)|^{2}=1$ for all $x$, where $u(x)$ is the function appearing in self product form. Then, $\left|L\left(x_{1}, x_{2}\right)\right|=$ $\left|u\left(x_{1}\right)\right|\left|u^{*}\left(x_{2}\right)\right|=1$ for all $x_{1}$ and $x_{2}$.

Proof of (iii) $\Rightarrow$ (i): It is a well-known fact that [32] if two random variables are fully correlated, then one must be $\kappa$ times the other, where $\kappa$ is some complex number. If we choose some point $x_{0}$, then $f\left(x_{0}\right)$ will be a random variable. Likewise, $f(x)$ can be considered a random variable parameterized by $x$.
Therefore, if $f\left(x_{0}\right)$ and $f(x)$ are fully correlated, then there is a function $\kappa(x)$ such that $f(x)=\kappa(x) f\left(x_{0}\right)$. Thus,

$$
\begin{aligned}
J\left(x_{1}, x_{2}\right) & =\left\langle f\left(x_{1}\right) f^{*}\left(x_{2}\right)\right\rangle \\
& =\left\langle\kappa\left(x_{1}\right) f\left(x_{0}\right) \kappa^{*}\left(x_{2}\right) f^{*}\left(x_{0}\right)\right\rangle \\
& =J\left(x_{0}, x_{0}\right) \kappa\left(x_{1}\right) \kappa^{*}\left(x_{2}\right) \\
& =u\left(x_{1}\right) u^{*}\left(x_{2}\right),
\end{aligned}
$$

where $u(x)=\sqrt{J\left(x_{0}, x_{0}\right)} \kappa(x)$.

The above proofs cyclically complete the proof of the equivalence of all five items stated at the beginning of this appendix.

Acknowledgment. H. M. Ozaktas acknowledges partial support of the Turkish Academy of Sciences. T. C. Gulcu acknowledges the support of TUBITAK through a graduate scholarship. We also would like to express our heartfelt thanks to Serdar Yüksel of Queen's University, Kingston, Canada, for his contributions to earlier stages of this research [24].

\section{REFERENCES}

1. J. Perina, Coherence of Light (Van Nostrand Reinhold, 1971).

2. M. Born and E. Wolf, Principles of Optics, 6th ed. (Pergamon, 1980).

3. J. W. Goodman, Statistical Optics (Wiley, 1985).

4. L. Mandel and E. Wolf, Optical Coherence and Quantum Optics (Cambridge University, 1995).

5. E. L. O'Neill, Introduction to Statistical Optics (Dover, 2003).

6. F. Gori, M. Santarsiero, R. Simon, G. Piquero, R. Borghi, and G. Guattari, "Coherent-mode decomposition of partially polarized, partially coherent sources," J. Opt. Soc. Am. A 20, 78-84 (2003).

7. J. Tervo, T. Setala, and A. T. Friberg, "Degree of coherence for electromagnetic fields," Opt. Express 11, 1137-1143 (2003).

8. E. Wolf, "Unified theory of coherence and polarization of random electromagnetic beams," Phys. Lett. A 312, 263-267 (2003).

9. T. Setala, J. Tervo, and A. T. Friberg, "Complete electromagnetic coherence in the space-frequency domain," Opt. Lett. 29, 328-330 (2004).

10. P. Réfrégier and F. Goudail, "Invariant degrees of coherence of partially polarized light," Opt. Express 13, 6051-6060 (2005).

11. P. Réfrégier and A. Roueff, "Coherence polarization filtering and relation with intrinsic degrees of coherence," Opt. Lett. 31, 11751177 (2006).

12. F. Gori, M. Santarsiero, and R. Borghi, "Maximizing Young's fringe visibility through reversible optical transformations," Opt. Lett. 32 , 588-590 (2007).

13. A. Luis, "Degree of coherence for vectorial electromagnetic fields as the distance between correlation matrices," J. Opt. Soc. Am. A 24, 1063-1068 (2007).

14. A. Luis, "Maximum visibility in interferometers illuminated by vectorial waves," Opt. Lett. 32, 2191-2193 (2007).

15. R. Martinez-Herrero and P. M. Mejias, "Maximum visibility under unitary transformations in two-pinhole interference for electromagnetic fields," Opt. Lett. 32, 1471-1473 (2007).

16. R. Martinez-Herrero and P. M. Mejias, "Relation between degrees of coherence for electromagnetic fields," Opt. Lett. 32, 1504-1506 (2007).

17. A. Luis, "Modulation of coherence of vectorial electromagnetic waves in the Young interferometer," Opt. Lett. 33, 1497-1499 (2008).

18. P. Réfrégier and A. Luis, "Irreversible effects of random unitary transformations on coherence properties of partially polarized electromagnetic fields," J. Opt. Soc. Am. A 25, 2749-2757 (2008).

19. K. Yamazoe, "Computation theory of partially coherent imaging by stacked pupil shift matrix," J. Opt. Soc. Am. A 25, 3111-3119 (2008). 
20. R. Martinez-Herrero and P. M. Mejias, "Maximizing Young's fringe visibility under unitary transformations for mean-square coherent light," Opt. Express 17, 603-610 (2009).

21. A. Luis, "Coherence and visibility for vectorial light," J. Opt. Soc. Am. A 27, 1764-1769 (2010).

22. K. Yamazoe, "Two matrix approaches for aerial image formation obtained by extending and modifying the transmission cross coefficients," J. Opt. Soc. Am. A 27, 1311-1321 (2010).

23. A. T. Friberg and J. Turunen, "Optics communications," Opt. Commun. 283, 4423-4426 (2010), special issue on Electromagnetic Coherence and Polarization.

24. H. M. Ozaktas, S. Yüksel, and M. A. Kutay, "Linear algebraic theory of partial coherence: discrete fields and measures of partial coherence," J. Opt. Soc. Am. A 19, 1563-1571 (2002).

25. M. A. Kutay, H. M. Ozaktas, M. F. Erden, and S. Yüksel, "Discrete matrix model for synthesis of mutual intensity functions," Proc. SPIE 4392, 87-98 (2001).

26. M. A. Kutay, H. M. Ozaktas, and S. Yüksel, "Synthesis of optical fields characterized by their mutual intensity functions," in Optical Information Processing: A Tribute to Adolf Lohmann, $\mathrm{H}$. John Caulfield, ed. (SPIE, 2002), pp. 103-122.

27. A. Luis, "An overview of coherence and polarization properties for multicomponent electromagnetic waves," in Advances in Information Optics and Photonics, A. T. Friberg and R. Dändliker, eds. (SPIE, 2008), pp. 171-188.

28. R. Horstmeyer, S. B. Oh, and R. Raskar, "Iterative aperture mask design in phase space using a rank constraint," Opt. Express 18, 22545-22555 (2010).

29. R. Horstmeyer, S. B. Oh, and R. Raskar, "View-dependent displays and the space of light fields," arXiv:1008.0034 (2010).

30. Z. Zhang, "Analysis and synthesis of three-dimensional illumination using partial coherence," Ph.D. thesis (Stanford, 2011).

31. R. Castañeda, "Electromagnetic wave fields in the microdiffraction domain," Phys. Rev. A 89, 013843 (2014).

32. A. Papoulis, Probability, Random Variables, and Stochastic Processes, 3rd ed. (McGraw-Hill, 1991).

33. A. W. Naylor and G. R. Sell, Linear Operator Theory in Engineering and Science (Springer-Verlag, 1982)

34. B. Zhang and B. Lu, "Transformation of Gaussian Schell-model beams and their coherent-mode representation," J. Opt. 27, 99-103 (1996).
35. T. Habashy, A. T. Friberg, and E. Wolf, "Application of the coherentmode representation to a class of inverse source problems," Inverse Prob. 13, 47-61 (1997).

36. B. E. A. Saleh and M. C. Teich, Fundamentals of Photonics (Wiley, 1991).

37. G. Strang, Linear Algebra and its Applications, 3rd ed. (Harcourt Brace Jovanovich, 1988)

38. M. J. Bastiaans, "New class of uncertainty relations for partially coherent light," J. Opt. Soc. Am. A 1, 711-715 (1984).

39. M. A. Alonso, "Radiometry and wide-angle wave fields III: partial coherence," J. Opt. Soc. Am. A 18, 2502-2511 (2001).

40. H. Lajunen, J. Tervo, and P. Vahimaa, "Overall coherence and coherent-mode expansion of spectrally partially coherent plane-wave pulses," J. Opt. Soc. Am. A 21, 2117-2123 (2004).

41. J. Tervo, T. Setala, and A. T. Friberg, "Theory of partially coherent electromagnetic fields in the space-frequency domain," J. Opt. Soc. Am. A 21, 2205-2215 (2004).

42. P. Vahimaa and J. Tervo, "Unified measures for optical fields: degree of polarization and effective degree of coherence," J. Opt. A. 6 , S41-S44 (2004).

43. K. Blomstedt, T. Setala, and A. T. Friberg, "Effective degree of coherence: general theory and application to electromagnetic fields," J. Opt. A. 9, 907-919 (2007).

44. A. Luis, "Overall degree of coherence for vectorial electromagnetic fields and the Wigner function," J. Opt. Soc. Am. A 24, 2070-2074 (2007).

45. P. Martinsson, H. Lajunen, and A. T. Friberg, "Communication modes with partially coherent fields," J. Opt. Soc. Am. A 24, 3336-3342 (2007).

46. H. Gamo, "Intensity matrix and degree of coherence," J. Opt. Soc. Am. A 47, 976 (1957).

47. H. Gamo, "Matrix treatment of partial coherence," in Progress in Optics, E. Wolf, ed. (North-Holland, 1964), Vol. 3, pp. 187-332.

48. K. Kim, D. Y. Park, and J. G. Kim, "Entropic measure of global coherence of a fluctuating field," J. Korean Phys. Soc. 35, 186-189 (1999).

49. S. Withington, M. P. Hobson, and R. H. Berry, "Representing the behavior of partially coherent optical systems by using over complete basis sets," J. Opt. Soc. Am. A 21, 207-217 (2004).

50. I. Csiszár and J. Körner, Information Theory: Coding Theorems for Discrete Memoryless Systems (Cambridge University, 2011). 